\title{
The First Stage of the "BTN-Neutron" Space Experiment onboard the Russian Segment of the International Space Station
}

\author{
V. I. Tret'yakov' ${ }^{1}$ I. G. Mitrofanov' ${ }^{1}$ Yu. I. Bobronitskii ${ }^{2}$, A. V. Vostrukhin ${ }^{1}$, N. A. Gunko , \\ A. S. Kozyrev' ${ }^{1}$, A.V. Krylov, M. L. Litvak ${ }^{1}$, M. Lopez-Alegria ${ }^{5}$, V. I. Lyagushin ${ }^{6}$, A. A. Konovalov', \\ M. P. Korotkov' ${ }^{2}$, P. V. Mazurov ${ }^{6}$, M. I. Mokrousov ${ }^{1}$, A. V. Malakhov ${ }^{1}$, I. O. Nuzhdin ${ }^{1}$, \\ S. N. Ponomareva ${ }^{2}$, M. A. Pronin 6 , A. B. Sanin'1, G. N. Timoshenko ${ }^{4}$, T. M. Tomilina ${ }^{2}$, \\ M. V. Tyurin ${ }^{7}$, A. I. Tsygan ${ }^{3}$, and V. N. Shvetsov ${ }^{4}$ \\ ${ }^{1}$ Space Research Institute, Russian Academy of Sciences, Moscow, Russia \\ ${ }^{2}$ Blagonravov Institute of Machine Science, Russian Academy of Sciences, Moscow, Russia \\ ${ }^{3}$ Ioffe Institute of Physics and Technology, Russian Academy of Sciences, St. Petersburg, Russia \\ ${ }^{4}$ Joint Institute of Nuclear Research, Dubna, Russia \\ ${ }^{5}$ Johnson Space Flight Center, NASA, Houston, Texas, USA \\ ${ }^{6}$ Korolev Rocket and Space Corporation "Energiya”, Korolev, Moscow oblast, Russia \\ ${ }^{7}$ Gagarin Center of Cosmonaut Training, Zvezdny, Russia
}

Received August 28, 2008

\begin{abstract}
The aims and tasks of the space experiment "BTN-Neutron" onboard the Russian segment of the International Space Station are described. The experiment deals with detection of fast and epithermal neutrons, X-rays, and gamma rays. Characteristics and a short description of scientific instrumentation BTN-M1 for this experiment are presented, as well as the first results of operation of the apparatus onboard the station during the first two years of flight.
\end{abstract}

DOI: $10.1134 / \mathrm{S} 0010952510040027$

\section{INTRODUCTION}

Investigation of neutron and gamma-ray background in the near-Earth space onboard manned and automatic orbital spacecraft has started since the first flights into space and is being continued at the present time. Such experiments were conducted and are carried out using both passive and active methods. In the first case measurements are made with passive detectors. Numerous experiments onboard the Mir orbital station $[1,2]$ and the SSNTD experiment onboard the International Space Station (ISS) [3] represent this type of experiments. The data of detectors are processed onboard or on the ground to give integral information on particle spectra during the exposure, but this method does not allow one to study time dynamics of neutron and gamma-ray fluxes as a function of solar activity level. The second, active method makes provision for carrying out onboard experiments using neutron and gamma-ray spectrometers with continuous detection of radiation fluxes and transmission of measurement data through a radio channel to the Earth. The experiments DOSMIR onboard the Mir station [4] and DOSTEL onboard the ISS [5] were made based on the second method. This method allows one to get considerably larger amount of useful scientific information, though it requires more complex instru- mentation. Real-time measurements of the neutron and gamma-ray background allow one to solve successfully the problems of radiation safety of the crew and instrumentation during long-term space flights. The space experiment "BTN-Neutron" whose first stage is in progress at the moment onboard the Russian segment (RS) of the ISS belongs to the type of active experiments.

This space experiment "BTN-Neutron" for measuring neutron fluxes at the near-Earth orbit in a wide range from thermal energies up to energies exceeding $100 \mathrm{MeV}$ was suggested as early as 1997 in the course of discussion of the scientific program for the ISS. According to its original concept, proposed by the Space Research Institute of Russian Academy of Sciences, the experiment was intended to detect neutrons in a wide energy range by several detectors of the instrumental complex with a general title BTN (Russian abbreviation for the Onboard Neutron Telescope). These measurements should provide for a complete set of data for physical interpretation and unambiguous identification of various components of the neutron radiation in the near-Earth space: neutron fluxes from the upper atmosphere, from the $I S S$ constructions, and from solar proton events (SPE). 
In 2001 NASA launched to Mars the Mars Odyssey mission for global exploration of the surface of the red planet from a circular polar orbit. The payload of this spacecraft included the complex of GRS gamma-ray spectrometer for studying the gamma-ray and neutron radiation of the Martian surface. The GRS complex consisted of a gamma-ray detector, based on cooled high-purity germanium, developed in Arizona State University, USA, of a neutron spectrometer for thermal and epithermal neutrons manufactured in the Los-Alamos National Laboratory, USA and of the Russian detector HEND (High Energy Neutron Detector) of high-energy neutrons developed in the Space Research Institute. Such measurements from a near-Mars orbit are possible due to the thin atmosphere of Mars, which freely lets cosmic rays passing to the planet surface, and also almost without absorption secondary neutrons and gamma-rays produced in the Martian soil are able to move away into space. In this case the spectral density of the flux of secondary nuclear radiation is determined by the elemental composition of the soil substance.

At the present time the HEND instrument continues successfully operate onboard the Mars Odyssey spacecraft, and, according to NASA decision, the measurements are planned to be prolonged at least up to October 2010. The data already obtained have demonstrated a high research potential of nuclear physics methods for studying the composition and structure of the upper layer substance of a celestial body bombarded by energetic particles of cosmic rays. Using the data of neutron measurements made by the HEND instrument and other instruments of the GRS complex it has been established that near-polar regions of Mars located higher than $60^{\circ}$ north and south latitudes represent permanent frosty soil with high content of water ice whose mass fraction can reach $50-70 \%$ of soil [6]. The data accumulated for more than three Martian years allowed one to investigate dynamics of seasonal processes of condensation of atmospheric carbonic acid snow on the surface of Mars [7]. The data of the HEND instrument also allowed one to estimate the contribution of the neutron component to radiation background at the Martian orbit under conditions of the quiet Sun and during SPEs. It was demonstrated that powerful SPEs could make the bulk contribution to the total annual radiation dose on the surface of Mars. Therefore, their forecasting and monitoring will be of primary importance for planning future interplanetary missions.

After launch of the Mars Odyssey mission, a spare flight unit of the HEND instrument has remained in SRI of RAS. It was suggested to use it as a detector unit of the BTN-M1 instrumentation for carrying out a first stage of the BTN-Neutron space experiment. Such an approach also allows one to make synchro- nous measurements on near-Earth and Martian orbits for simultaneous stereoscopic observation of the solar activity and for studying the neutron background variations.

Scientific instrumentation BTN-M1 was designed and manufactured in SRI of RAS with participation of Special Design Office of SRI, Blagonravov Machine Building Institute of RAS, Joint Institute of Nuclear Research, and RAS Institute of Medical and Biological Problems. Training of crew actions on installing the detector unit on the outer surface of Zvezda module of Russian segment of the $I S S$ during extra-vehicle activity (extraVA) was performed in the Gagarin Cosmonaut Training Center. Apparatus control, acquisition and storing of primary telemetry and guidance data is made by the Flight Control Center of TsNIIMaSh.

Assembling the research instrumentation onboard the ISS by the crew members of the 14th basic expedition was the most important concluding stage of the experiment preparation. First, this work included installing the electronic block BTN-ME inside the module Zvezda and laying internal cables for connection to service systems of the station. Second, during two exits of the $I S S$ crew into open space the detection block was installed on the outer surface of the Zvezda module and connected through hermetically sealed sockets to the electric circuits of the ISS Russian Segment. Because of the tight schedule of the extaVA during the first exit on November 23, 2006 astronauts succeeded only in fixing mechanically the detection block and connecting the power supply cable. It should be noted that the crew proposed the solution of making this connection under critical conditions of substantial over-duration of scheduled staying in the open space. This proposal was accepted by the control group in the FCC-M. Because of this solution the power supply for the automatic system for thermal regime provision (ASTRP) was ensured, which allowed one to avoid its failure due to overcooling in the switched-off state. During the second exit on February 22, 2007 the crew successfully completed connection of the second command-telemetry cable. After that, the instrumentation was switched on by a command from the ground.

At present, the research instrumentation BTN-M1 is connected with systems of power supply and telemetry ensuring scientific measurements in the standard mode with everyday transmission of data and service information to the ground.

\section{NEUTRONS IN THE NEAR-TERRESTRIAL SPACE}

Neutrons at a height of the ISS are known to be produced in three physical processes. First, energetic 
protons and ions of galactic cosmic rays and from the radiation belts of the Earth's magnetosphere generate neutrons with energies of about 10-20 MeV due to nuclear reactions in the upper atmosphere of the Earth. Thus produced secondary neutrons are slowed in the atmosphere matter, a part of them being absorbed in nuclear reactions of capture to form new nuclei. Another part is decayed, but a substantial fraction leaves the atmosphere [8], forming in the nearterrestrial space the so-called neutron albedo of the Earth. The flux and energy spectrum of albedo neutrons are variable in time and space, since they depend on the flux and energy of charged particles in the magnetosphere, as well as on density, temperature, and composition of the upper layers of the atmosphere.

Second, around the ISS the proper induced neutron emission is produced due to interactions of of energetic charged particles of the magnetosphere with the matter of the station itself $[9,10]$. These local neutrons fluxes are present around the station in the region with a characteristic scale of the order of its dimensions, and they are also variable along the station orbit due to irregularity of the flux of charged particles.

The third cause of the existence of neutrons in the near-terrestrial space is the solar activity. It is well known that during some powerful SPEs the fluxes of high-energy neutrons are generated in active regions on the Sun [11]. The neutron lifetime is about $15 \mathrm{~min}$, which is comparable to the time of flight of relativistic particles from the Sun to the Earth's orbit. Therefore, a substantial fraction of high-energy solar neutron can reach the vicinity of the Earth.

Solar neutrons were detected for the first time during the solar flare on June 21, 1980 using the GRS instrument installed on the NASA research satellite Solar Maximum Mission (SMM) [12]. Later on, solar neutrons were recorded by the same instrument on the $S M M$ [13], by the SONG instrument on the Coronas- $F$ spacecraft [14], and in joint measurements of groundbased neutron monitors and instruments of the Integral space observatory [15]. The energy spectra and fluxes of neutrons near the Earth were measured, and the estimates of the total neutron flux generated in the active region on the Sun were made. These estimates are of great importance especially for the reason that fluxes of the neutral particles (neutrons and gammarays) are not distorted by the interplanetary magnetic field, thus reflecting the processes of acceleration of charged particles up to relativistic energies in the active regions of solar flares.

One should take into account that during EPS the proton component of cosmic rays increases substantially (by orders of magnitude). Accordingly, the fluxes of secondary neutrons from the Earth's atmosphere and from the station itself also increase. Therefore, in order to estimate the fraction of solar neutrons in the total number of recorded particles, it is necessary to analyze in detail the contributions of all three sources of neutron radiation in the near-terrestrial space: the Earth's atmosphere, the station's matter, and active regions of the Sun.

\section{GOALS OF THE EXPERIMENT AND EXPECTED RESULTS}

\subsection{Basic Goals of the Experiment}

The BTN-Neutron experiment has the following basic goals.

1) Construction of the map of secondary neutron radiation of the upper atmosphere of the Earth taking into account the effects of longitude and latitude of the measurement point, time of the day, and the state of the magnetosphere and upper atmosphere.

2) Measurement of the neutron component of solar radiation during SPEs.

3) Construction of a physical model of the local neutron background from the ISS under different conditions of its orbital flight.

There are some additional goals of the experiment on its first stage. They are as follows.

4) Synchronous measurements by the HEND instrument in the Martian orbit and by BTN-M1 in the near-terrestrial orbit of the effects of increased neutron component of the space radiation background in the vicinity of Mars and the Earth during SPE.

5) Detection of cosmic gamma-ray bursts near the Earth in order to participate in the program of determining the direction to a flashed source of gamma-ray emission by the method of interplanetary triangulation.

6) Investigation of radiation resistance of the samples of new scintillation materials in order to estimate possibilities of using them for detection of gamma rays and neutrons in future space experiments.

\subsection{Expected Results of the First Stage of the Experiment}

It is suggested in the course of performing the first stage of the BTN-Neutron experiment to carry out the following research studies and to obtain their practical results.

1) Investigation of the secondary neutron emission generated in the upper atmosphere of the Earth under the action of GCR as a function of longitude and latitude of the place of observation, hour of the day, state of the atmosphere, and characteristics of charged particle fluxes in the radiation belts. Study of the effect of increased neutron background in the region of the South Atlantic Magnetic Anomaly (SAMA) and at high geomagnetic latitudes for different phases of the 
solar cycle from the minimum in 2007-2009 years to the maximum in 2012-2013.

2) Construction of a model of neutron generation in active regions of the Sun based on comparison of the results of measuring the fluxes of high-energy neutrons with the data of measurements of $x$-ray and gammaray emissions, and detection of energetic protons by other spacecraft.

3) Creation of a prompt model of neutron environment onboard the ISS in order to control radiation conditions and to elaborate recommendations for minimization of the radiation dose rate for the crew.

4) Based on the BTN-Neutron and HEND data, comparison, of the neutron component of the radiation background in the near-terrestrial orbit and at orbit around Mars in order to make a forecast of neutron contribution to the radiation environment onboard an interplanetary vehicle in different periods of solar activity.

5) Provision of the data about detection of cosmic gamma-ray bursts for the international program of interplanetary triangulation and determination of coordinates of burst sources on the celestial sphere.

6) Investigation of the effects of radiation damage of new scintillation crystals $\mathrm{LaBr}_{3}: \mathrm{Ce}^{3+}, \mathrm{LaCl}_{3}: \mathrm{Ce}^{3+}$, $\left(\mathrm{Lu}_{0.5} \mathrm{Y}_{0.5}\right) \mathrm{AlO}_{3}: \mathrm{Ce}^{3+},\left(\mathrm{Lu}_{0.7} \mathrm{Y}_{0.3}\right) \mathrm{AlO}_{3}: \mathrm{Ce}^{3+},\left(\mathrm{Lu}_{2} \mathrm{SiO}_{5}\right)$ : $\mathrm{Ce}^{3+},\left(\mathrm{YAlO}_{3}\right): \mathrm{Ce}^{3+},(\mathrm{LuAl}) \mathrm{O}_{3}: \mathrm{Ce}^{3+}$, and $(\mathrm{LuY}) \mathrm{SiO}_{5}$ : $\mathrm{Ce}^{3+}$ under space flight conditions in order to estimate their applicability in advanced space detectors of gamma rays and neutrons, including those for subsequent stages of the space experiment BTN-Neutron.

\section{DESCRIPTION OF INSTRUMENTATION BTN-M1}

\subsection{Composition of the BTN-M1 Instrumentation}

The BTN-M1 scientific equipment includes two units: detector assembly BTN-MD-MF with overall dimensions $594 \times 462 \times 220 \mathrm{~mm}$, a mass of $11.5 \mathrm{~kg}$, and maximal power consumption $14.7 \mathrm{~W}$ placed on the outer surface of the Zvezda module and the internal electronic block BTN-ME with dimensions $255 \times$ $265 \times 115 \mathrm{~mm}, 3.4 \mathrm{~kg}$ mass, and maximal power consumption $3.4 \mathrm{~W}$ (Fig. 1).

The instrumentation also includes a set of cables for connecting equipment inside and outside the station with a mass of about $14.4 \mathrm{~kg}$; two cassettes with dimensions $110 \times 140 \times 195 \mathrm{~mm}$ and masses $1.9 \mathrm{~kg}$ and $0.8 \mathrm{~kg}$, respectively, for placing spare electronic cards of the electronic unit and for packing boards with dosimeters and samples of scintillators to be returned to the ground after their exposure in orbit. The total mass of BTN-M1 equipment is more than $30 \mathrm{~kg}$. The total power consumption in the measurement mode is about $18 \mathrm{~W}$.

\subsection{Construction of the Outer Detector Assembly BTN-MD-MF}

The detector unit BTN-MD is installed inside a box-shaped frame BTN-MF which serves several functions.

First, the frame BTN-MF ensures the optimal thermal regime for the BTN-MD unit at different illumination conditions onboard the ISS. In order to transfer a released heat from the BTN-MD unit to the BTN-MF frame, copper bars with a cross-section of $30 \mathrm{~mm}^{2}$ are used. The frame itself has for heat release two oppositely located emitting radiators.

The ASTRP of the BTN-MD unit has individual onboard power supply and operates independent of other electronic circuits of the instrument. The ASTRP is tuned to switch internal heater on and off when temperature of the BTN-MD unit body is equal to $-23^{\circ} \mathrm{C}$ and $-18^{\circ} \mathrm{C}$, respectively, which allows one to keep the temperature of electronic circuits in the range higher than $-23^{\circ} \mathrm{C}$. An additional heater with a power of $6 \mathrm{~W}$ is set in the BTN-MD unit. It is activated by a discrete command from the ground and used under conditions of strong overcooling of the unit, when heat released by the ASTRP cannot provide for the admissible thermal regime of the instrument operation.

In order to reduce heat exchange with the external handrails of the Zvezda module on which the detector assembly BTN-MD-MF is mounted, special heatinsulated bridge sleeves are installed on matching sites of the BTN-MF frame. The assembly of BTN-MD unit and BTN-MF frame is covered by multilayer shield vacuum heat insulation (SVHI), excluding radiators, matching sites for docking with the adapter platform, and electric sockets. By this means, the external detector assembly is completely heat-insulated from the ISS and has an autonomous system of thermal regulation. In order to avoid overcooling of the BTN-MD unit at the stage of installing it during extraVA, both radiators of the frame are temporarily protected by heat-insulating jackets from SVHI. The jackets are removed by the crew, when the detector unit has been installed to its place, and power supply has been connected. In order to control the temperature regime of the BTN-MF frame, two thermal sensors TP-18-05 are arranged on one of its radiators and on the matching site. Their data are transmitted to the Earth as a part of service information practically at every orbit.

Numerical simulation of the operation of the external detector thermal conditions was performed at the stage of designing the equipment for various conditions of illumination, and the obtained results were checked at the stage of testing the instrument in a thermal vacuum chamber of the check-out stand of Space Research Institute of RAS. The data of measurements 


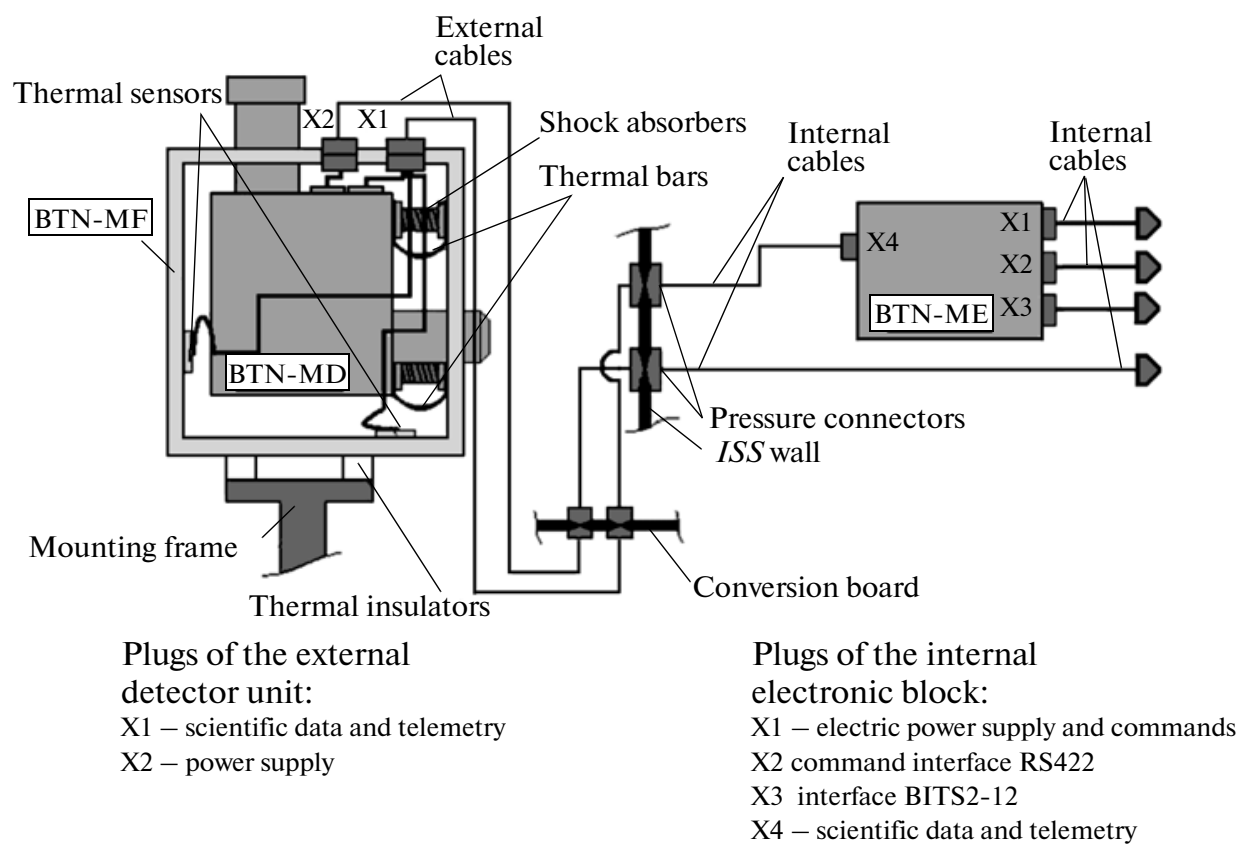

Fig. 1. Block diagram of scientific equipment.

after the beginning of flight tests of the BTN-M1 instrument confirmed the validity of technical solutions chosen to provide for an optimal thermal regime of the unit at all flight modes of the ISS.

Second, the BTN-MF frame also ensures protection of the detector and electronic units against various mechanical impacts. For example, in connection with necessity of damping the vibrations on the ISS board that could result in electric noise due to microphone effect in photomultipliers, the BTN-MD unit is mounted inside the BTN-MF frame using four special mechanical shock absorbers developed in collaboration with the Blagonravov Institute of Machine Science of Russian Academy of Sciences (Moscow).

Third, electric plugs X1 and X2 of the RS50 type are arranged on the BTN-MF frame. Cables from plugs of the BTN-MD unit and from thermal sensors are connected to them from inside. The X1 plug serves for transmitting scientific and telemetry data to the BTN-ME unit and for receiving the commands of control from it. The $\mathrm{X} 2$ plug is designed to provide power supply to electronic circuits and ASTRP (Automatic system of Thermal Regulation Providing) of the BTN-MD unit. The BTN-MF frame has also a metalized bridge that is fixed to a support arm, thus providing for ground connection of the external detector unit to the station's mainframe through locks of the adapter platform.

In order to accomplish scientific tasks it was required that the BTN-MD unit at standard installation would be oriented in such a way that the axis of unit scintillator should be directed to zenith. One should add to this the requirements of storing the instrument temporarily inside the $I S S$, possibility of mounting and dismantling the equipment while working in a space suite, and securing access to the handrails after installation of the unit. Taking these requirements into account, designers of the Rocket-Space Corporation "Energiya" have developed a special assemblage-and-installation device on which the BTN-MD-MF assembly was installed. This device included the support arm, adapter platform, and three quick-detachable locks.

\subsection{The Unit BTN-MD for Detection of Neutrons and Gamma Rays}

The detector unit BTN-MD is a spare flight specimen of the HEND instrument (Fig. 2). This instrument is a spectrometer with four independent neutrons detectors. Three detectors of epithermal neutrons (in Fig. 2 they are designated as SD, MD, and LD) represent proportional gas counters filled with ${ }^{3} \mathrm{He}$, while the fourth detector of neutrons (marked in Fig. 2 as SC) is manufactured from stilbene $\mathrm{C}_{14} \mathrm{H}_{12}$ surrounded by active anticoincidence shield of $\mathrm{CsI}: \mathrm{Tl}^{3+}$ crystal.

Neutrons in the counters based on ${ }^{3} \mathrm{He}$ are detected through the reaction of capture with production of a proton and a tritium nucleus (triton):

$$
n+{ }^{3} \mathrm{He} \longrightarrow p+{ }^{3} \mathrm{H}+764 \mathrm{keV},
$$




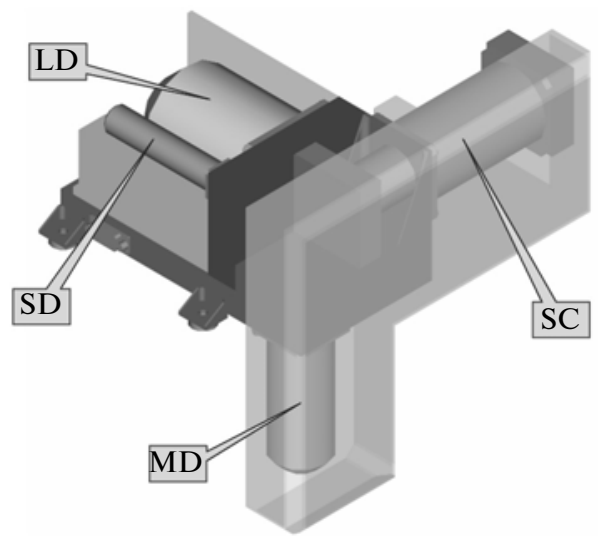

Fig. 2. Structure chart of the BTN-MD unit.

the products of this reaction being detected due to ionization caused by them. The energy of $764 \mathrm{keV}$ released in this reaction is divided between the triton and proton in the proportion $1: 3$, i.e., the triton carries away $191 \mathrm{keV}(25 \%)$, and the proton carries away the energy of $573 \mathrm{keV}(75 \%)$. If the reaction occurs near the counter wall, the particles can leave the detecting volume, only a part of the total energy being released in the counter. Therefore, when neutrons are detected by a ${ }^{3} \mathrm{He}$ counter, the spectrum of energy release has a characteristic shape with two peaks beginning from $191 \mathrm{keV}$ (the reaction threshold) and at energy of $764 \mathrm{keV}$ (the complete absorption peak). The cross-section of capture reaction (1) increases in inverse proportion to the relative velocity of a neutron and ${ }^{3} \mathrm{He}$ nucleus. Therefore, the efficiency of neutron detection increases with decreasing energy of neutrons, and it becomes maximal for thermal neutrons.

Signals from the counter come to the input of a charge-sensitive preamplifier and then to an analogdigital converter. Measurement of the energy spectrum of reaction (1) allows one to separate reliably the events associated with neutron detection from the contribution of charged particles and/or electric noise.

Three detectors have polyethylene shells of different thickness, which provide different degree of moderation for incident neutrons down to thermal energies at which their detection is most efficient. Each counter is also surrounded by a shield from cadmium foil that does not virtually let neutrons with energies $<0.4 \mathrm{eV}$ due to a very large absorption by cadmium. The SD detector with a polyethylene shell $2 \mathrm{~mm}$ thick has a maximum of sensitivity in the neutron energy range from $0.4 \mathrm{eV}$ up to $1 \mathrm{keV}$. The MD detector with polyethylene moderator of $15 \mathrm{~mm}$ thickness has the range of maximum sensitivity for neutrons with energies from $10 \mathrm{eV}$ to $100 \mathrm{keV}$. The thick double moderator of the LD detector with a thickness of $30 \mathrm{~mm}$ pro- vides for the maximum sensitivity in the energy range from $10 \mathrm{eV}$ up to $1 \mathrm{MeV}$.

The fourth detector of neutrons represents a scintillator based on a stilbene $\left(\mathrm{C}_{14} \mathrm{H}_{12}\right)$ crystal. This detector serves for detection of high-energy neutrons in the range from $400 \mathrm{keV}$ to $10 \mathrm{MeV}$ in 16 spectral channels. Stilbene is an organic scintillator crystal with a high content of hydrogen. When detecting a neutron with energy $E_{n}$, a recoil proton with energy $E_{p}<E_{n}$ is knocked out from the crystal lattice. The proton produces a scintillation flash whose intensity is proportional to the particle energy (signal SC/IN/N), and the flash is detected by a PM tube of the Hamamatsu R1924 type. Absorption of gamma rays can occur in stilbene as well, with production of a free electron which also produces a flash (signal $\mathrm{SC} / \mathrm{IN} / \mathrm{G})$. When detecting protons and electrons, the shapes of electric pulses from PM tubes are essentially different [16]. A special electronic scheme records this difference allowing one to distinguish these two classes of events with an error not exceeding $10^{-3}$. The outer scintillator ensures operation of a coincidence scheme thus protecting the inner detector from recording external charged particles. The outer detector is manufactured from the CsI : $\mathrm{Tl}^{3+}$ crystal which has a high density and large atomic number, representing an effective detector for charged particles with energies higher than $50 \mathrm{keV}$ [17]. The detection in stilbene of a count produced by an external proton is accompanied by a signal of detection of a charged particle in the outer scintillator. Based on this signal, counts of cosmic ray protons are excluded from the set of counts of neutron events. Flashes in this detector (signal SC/OUT) are detected by the PM tube of the Hamamatsu R1840 type.

Thus, at standard operation and basic settings of the logical scheme the detection unit BTN-MD generates six separate signals from five detectors: three 
signals about detection of epithermal neutrons in proportional counters $\mathrm{SD}, \mathrm{MD}$, and $\mathrm{LD}$; signal $\mathrm{SC} / \mathrm{IN} / \mathrm{N}$ about detection of high-energy neutrons in the inner scintillator SC/IN; signal SC/IN/G about detection of gamma rays in the same scintillator; and signal SC/OUT about detection of photons and/or charged particles in the outer scintillator. Complete processing of all above signals is performed in the BTN-MD unit: from primary amplification and analog-digital conversion in 16 spectral channels up to formation of the frames with scientific and telemetry data to be transmitted to the BTN-ME unit. For a time of accumulation of an individual frame six summed 16-channel spectra of counts are formed in the unit for every signal. Duration of the accumulation time of the spectra is determined by a command from the ground and can vary from 1 to 256 seconds.

The logic of a flash cell is also realized in the BTN-MD detection unit, which allows one, during accumulation of a current frame, to record two integral profiles of signals SC/IN/G (with a time resolution of $1.0 \mathrm{~s}$ ) and SC/OUT (with a resolution of $0.25 \mathrm{~s}$ ). In the case, when the current counting rate of these signals exceeded the background counting rate by a factor preset by command, these profiles are added to a frame of scientific information. The instrument can also be used in a regime when the profiles are recorded at formation of every frame, independent of the counting rate.

The BTN-MD unit has only internal time. The data of measurements are referenced to the ISS onboard time in the BTN-ME unit which every five minutes synchronizes the instrument's internal time using the time marker received from the onboard unit of the payload server. Three modes of operation are specified by logic of the unit operation: on-duty, basic research, and shortcut research with a veto put upon detection of gamma ray bursts. In the on-duty mode no scientific measurements are performed. The unit's electronic circuits execute the received commands, make measurements of temperature at six points of the instrumentation, and send frames with service information to the telemetry system. In the basic research mode, which is also the mode of operation by default, the BTN-MD unit forms the frames with scientific data, including all six physical signals and gamma-ray profiles with time resolutions of $1.0 \mathrm{~s}$ and $0.25 \mathrm{~s}$. The duration of accumulation of aggregate spectra of counts for each signal (duration of forming a separate frame) is determined by periodicity of injection of synchronization signal from BTN-ME to the BTN-MD unit. Upon reception of this signal, the logic block of the BTN-MD unit terminates accumulating the spectra and recording the profiles of counts, transmits the formed frame to the BTN-ME unit, and begins recording a new frame.
In the third shortcut mode, all six physical signals are also measured, but no profiles of gamma ray signals are recorded, which ensures some economy in the use of telemetry resources on the station. This mode is used when there is a limitation on the volume of daily transmitted data. The complete technical description of the instrument's electronics and software is presented in [18].

\subsection{Electronic Unit BTN-ME}

The BTN-ME electronic unit includes electronic boards designed for matching electric interfaces of the BTN-MD unit that obey the requirements of the Mars Odyssey spacecraft and the interfaces of electric systems of the ISS Russian segment. The external interfaces of the BTN-ME block provide for power supply, transmission of control commands and telemetry, and translation of time synchronization signals from the $I S S$ systems. The internal interfaces provide for power supply, transmission of control commands, reception of the frames of scientific data, and synchronization of frame formation for the BTN-MD unit.

The following functional tasks are run by the BTN-ME unit.

1. Generation and transmission of a logical signal SYNC, according to which the recording of the preceding frame of measurements in the detector unit BTN-MD is terminated, this frame is read out, and recording of the next frame begins. The time interval between successive signals SYNC can be set by a digital command within the limits from 1 to $256 \mathrm{~s}$. The choice of this interval determines the instrument's time resolution (accumulation time) and the amount of scientific data.

2. Readout of scientific data from the external unit BTN-MD, storage and transmission of telemetry data to BITS2-12 in the mode of direct transmission (DT) to the ground. The internal memory of the BTN-ME unit is $1 \mathrm{MB}$. When the time of one measurement is set to be $1 \mathrm{~s}$ (this corresponds to 1 -s periodicity of sending SYNC), the total daily amount of data (including the scientific and service data) is about 2.7 MB. In order to ensure uninterrupted measurements, one needs in this case to have at least 3 DT sessions per day. The mode of measurements, at which the accumulation time is equal to $60 \mathrm{~s}$, is optimal for operation with the ISS RS systems. In this case the total time of discharging the daily data of about 1 MB through the BITS2-12 systems of transmission is of order of $3 \mathrm{~min}$.

3. Reception and execution of discrete commands coming from the onboard BITS2-12 system and reception of digital commands through RS-422 interface coming from the payload server controlling scientific instrumentation of the ISS RS. A part of these commands is transmitted for execution to the external 
unit, the other commands are executed by the BTNME unit itself.

4. Arrangement of special boards with passive detector assemblies (PDAs) including crystals for advanced scintillation detectors and passive dosimeters. These PDAs are used at the first stage of the space experiment for studying radiation resistance of the scintillators and estimating their applicability to design instrumentation for other space experiments, including that for subsequent stages of the BTN-Neutron space experiment.

Electric plugs $\mathrm{X} 1-\mathrm{X} 4$ of the RS50 type are placed on the frame of BTN-ME, and cables from the ISS RS onboard systems are connected to them from inside. The X1 plug serves for power supply, through X2 the digital commands and time synchronization signals are received from the payload server, the $\mathrm{X} 3$ plug serves for transmission of discrete commands and telemetry data to BITS2-12. The plug X4 realizes communication with the external detector unit, including reception of scientific and telemetry data and transmission of control commands.

\section{INSTRUMENTATION CONTROL AND DATA TRANSMISSION}

Commands of two types control the BTN-M1 instrumentation: discrete (relay) and digital commands. The BTN-MD detector unit is controlled by internal digital commands through the RS-422 interface. These commands are generated and recieved from the electronic block BTN-ME come from there. This block is controlled by external digital commands (also through RS-422 interface) from the payload server and by external discrete commands from the System of Onboard Automation Control (SOAC) of the ISS RS. The external digital commands change the mode of BTN-MD operation, set measurement parameters (thresholds of the discriminator, levels of high voltage on the detectors, accumulation time, logic of anticoincidence, etc.), determine the operation conditions for ASTRP, and so on. The external discrete commands such as "Switch on BTN-ME", "Switch off BTN-ME", "The 1st half-set", "The 2nd half-set", "Data array discharge", "End of data array discharge" serve for controlling the BTN-ME unit and for putting the BTN-MD unit into a research mode under conditions, when it is impossible to send digital commands from the payload server to it, or when it is necessary to make diagnostics of operation of the unit's logical digital interface in emergency situations. When it is impossible to use digital commands for conversion of the BTN-MD unit into the research mode and its adjustment, provision is made for a possibility of switching it on in the shortcut research mode with default settings using a cyclogram of discrete commands.

The BTN-M1 instrumentation uses the standard telemetry system BITS2-12 of the ISS for transmission to the ground of data measurements. The frames with scientific and service data are accumulated in the 1 -MB native storage of the BTN-ME and are transmitted to BITS2-12 and next to the ground in radio communication sessions. Depending on the mode of operation of instrumentation the structure and size of a single telemetric frame with scientific and service data are variable. In the on-duty mode the size of a frame of BTN-MD is 56 bites; the research mode with permanent detection of bursts corresponds has the frame size 512 bits; and in the shortcut research mode this size id equal to 212 bites. When one sets the accumulation time equal to $60 \mathrm{~s}$, the total data stream is equal to $160 \mathrm{kB}$ per day and $850 \mathrm{kB}$ per day for the onduty mode and basic research mode, respectively. The electronic block BTN-ME adds to every frame formed by BTN-MD its own heading with a telemetry size of 64 bites. These additional data include temperatures of the BTN-MF frame and inside the BTN-ME unit, as well as service data about the state of the latter.

\section{MATHEMATICAL MODELING OF DETECTORS AND PHYSICAL CALIBRATIONS}

When developing the BTN-MD unit, numerical calculations based on the Monte Carlo method were made in order to evaluate the sensitivity of neutron detectors. In this mathematical simulation, carried out with participation of the Ioffe Physical-Technical Institute and Joint Institute of Nuclear Research (JINR), both self-made programs and commonly used MCNPX code distributed by the Oak Ridge National Laboratory, USA (http://rsicc.ornl.gov) were used. The aim of simulation was to construct response functions of the neutron detectors in the entire energy range from the cadmium threshold $0.4 \mathrm{eV}$ up to $10 \mathrm{MeV}$ and to compare the results of numerical calculations with experimental values that had been obtained for separate values of neutron energy. Figure 3 presents calculated curves of the response functions for proportional counters and stilbene scintillator. One can see that the instrument detects neutrons in a wide energy range from the cadmium threshold (about $0.4 \mathrm{eV}$ ) to energies higher than $10 \mathrm{MeV}$ with sufficiently high efficiency.

In order to verify the calculations, physical calibrations of the instrument's detectors were carried out in the Laboratory of Neutron Physics of JINR (Dubna). These calibrations included both measurements with the use of isotope neutron sources based on $\mathrm{Cf}^{252}$ and $\mathrm{Pu}-\mathrm{Be}$ and measurements with the neutron beam 


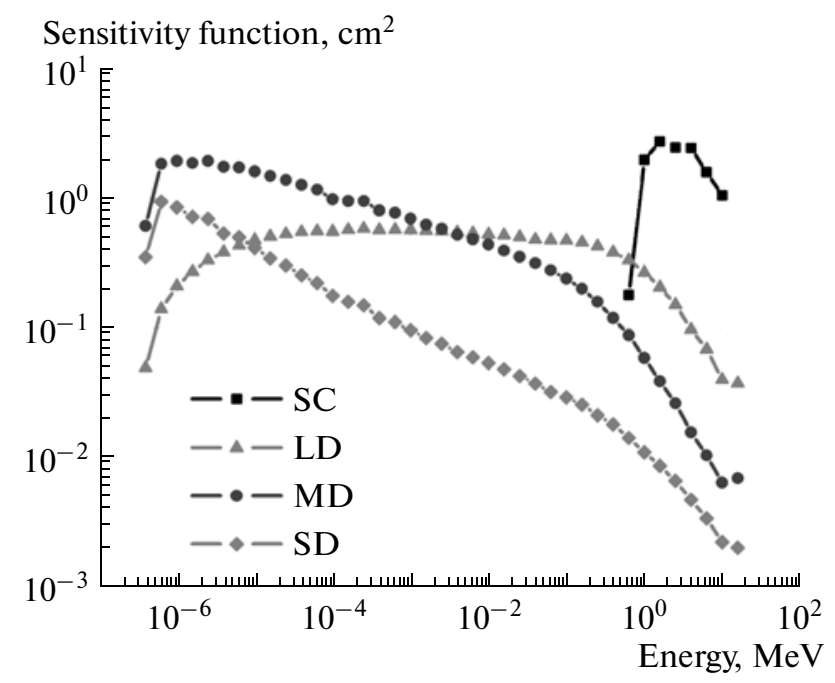

Fig. 3. Calculated curves of sensitivity functions for neutron detectors.

from a lithium target on the linear accelerator of protons. In the last case the neutron energy was determined by geometry of scattering of protons by lithium. For different angles it was equal to 100,500 , and $900 \mathrm{keV}$. The detector was installed on the neutron bean axis at distances $50 \mathrm{~cm}$ and $1.5 \mathrm{~m}$ from the lithium target. During all measurements the incident flux of neutrons was measured by a neutron monitor. Figure 4 presents a comparison of calculated curves with physical measurements for proportional counters and stilbene scintillator.

\section{START OF THE SPACE EXPERIMENT AND FIRST RESULTS}

\subsection{Primary Processing of Measurement Data}

The instrumentation was first activated on February 26,2007 . The mode of shortcut research measurements with default settings was switched on by discrete commands. The time reference was made according to the time of switching on the detector unit. At present, according to the telemetry data, electronic circuits of the detector and electronic units operate in the normal mode, and temperatures of both the units are within the admissible limits.

The scientific data of the space experiment BTNNeutron transmitted from the ISS board are stored in the database of the Information Retrieval System and databases of space experiments of the ISS RS in the Flight Control Center FCC-M. Associated data of navigation and ballistic support are also stored there, as well as the data of service telemetry coming to the FCC via independent communication channels.

At the moment, taking into account losses of information during transmission of the data to the ground,

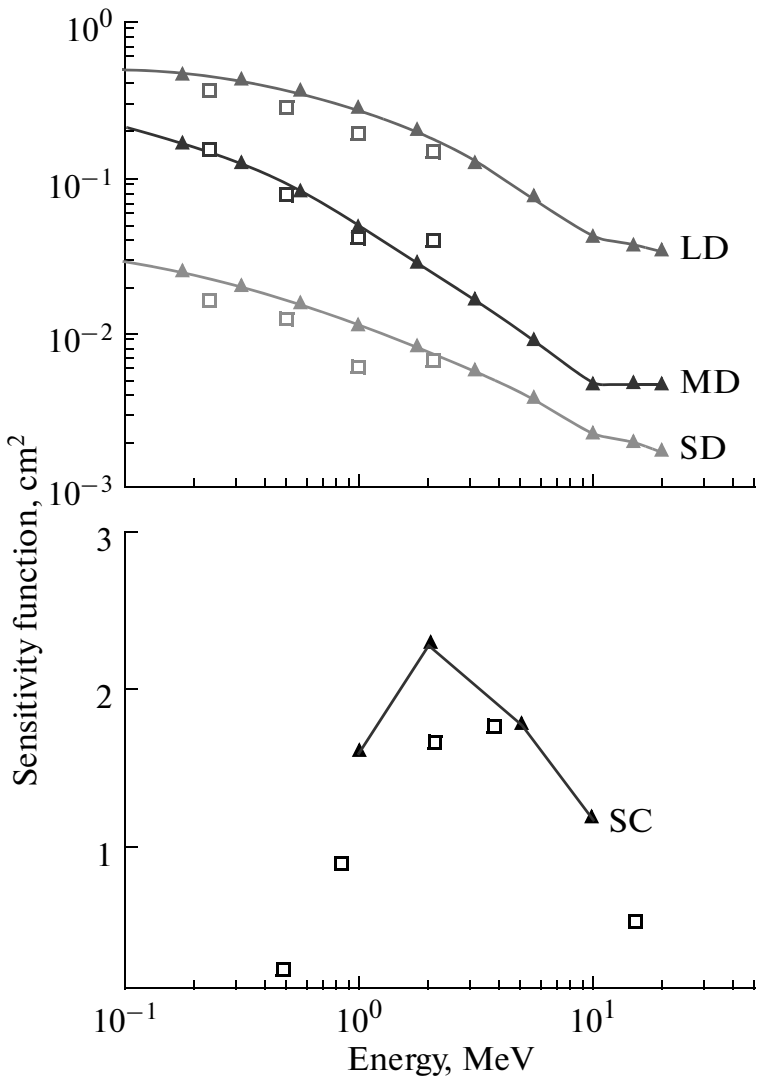

Fig. 4. Comparison of calculated response function curves with results of experimental calibrations for proportional counters (upper plot) and stilbene scintillator (lower plot). Squares and triangles show the data of measurements and calculated curves, respectively.

the total information content (scientific data and telemetry) without failures exceeds $90 \%$. From the FCC-M the entire information of the BTN-M1 instrumentation is copied to the database of the BTNNeutron experiment in the Space Research Institute. The mean monthly amount of the telemetry and scientific data varies from 40 to $60 \mathrm{MB}$.

The coordinates are referred based on the calculations of orbits parameters of the ISS in the geodetic coordinate system according to two-line elements of format TLE NORAD presented by NASA for public access through the Internet [http://www.spacetrack.org/tle_format.html] which are updated once per day using the data of radar measurements. Since the fluxes of neutron and gamma ray emission are anisotropic in the vicinity of the ISS, the data on ISS current orientation are included in accompanying information. These data are generated by the RS navigation system. Orbital and orientation parameters for short segments of the orbit above ground-based information points are recorded by the tracking service of FCC-M several times per day in the form of asset of ISS coordinates, components of the velocity vector, 


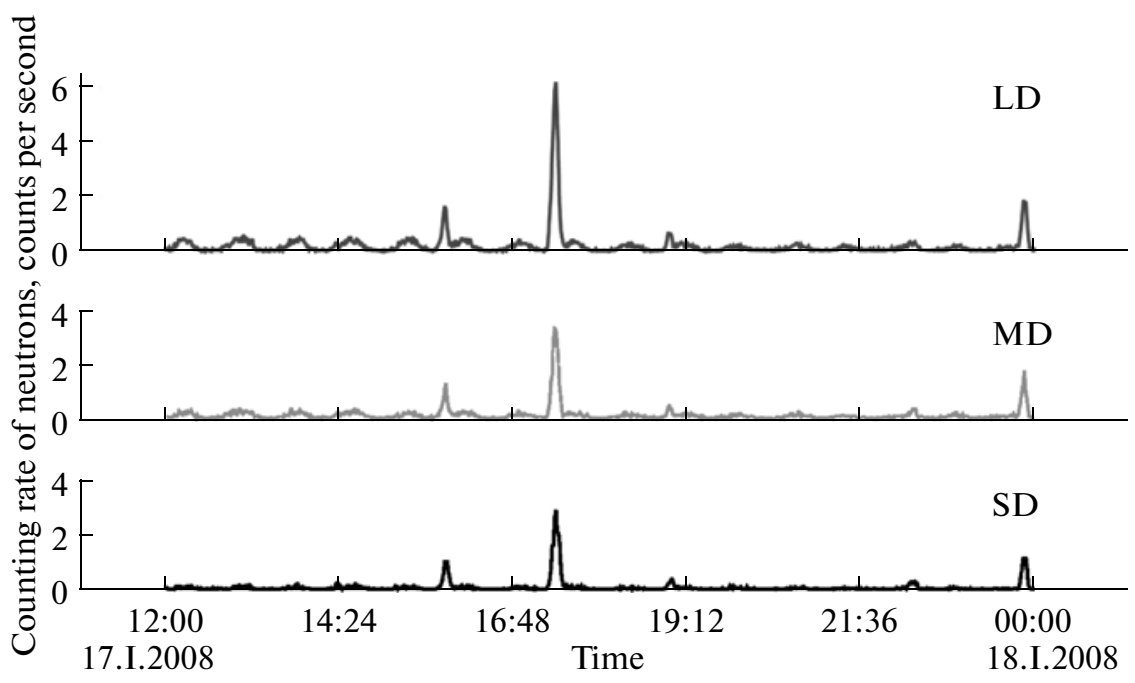

Fig. 5. Time profiles of neutron counting rate for proportional counters.

orientation quaternion, and onboard calendar time in seconds from January 6, 1980. They are copied into the experiment's databases in FCC-M and SRI, extrapolated to the starting moment of a given frame of measurements, and ascribed to it in the SRI database.

Primary processing of the data includes their representation in the form of profiles of the counting rates of neutrons and gamma rays, and instrumental spectra of counts. Coordinate maps of the background of neutron and gamma ray emission in the vicinity of the ISS are constructed by way of referring the profiles to the ISS coordinates.

\subsection{First Scientific Results}

Three basic scientific products of the space experiment were created at its first stage:

- uninterrupted sequence of instrumental spectra of counts in all detectors of the instrument with an exposure time of $60 \mathrm{~s}$, which is synchronized with the universal time;

- uninterrupted profiles of integral counting rates in all detectors of the instrument with a resolution of $60 \mathrm{~s}$, with reference to the universal time; and

- maps of counting rates in the neutron and gamma ray detectors in the geographic coordinates with spatial resolutions of $5 \times 5$ degrees in longitude and latitude.

No manifestations of solar activity were observed in the entire period 2007-2008 of performing the experiment, therefore, all the profiles of counting rate variability represented only variations of the background environment along the $I S S$ orbit. Figure 5 presents an example of the time profiles of counts for detectors
$\mathrm{SD}, \mathrm{MD}$, and LD for one-day time interval on January 17, 2008.

The variable character of the profile with a period close to the $I S S$ orbital period is clearly seen. The profiles of signals from fast neutrons and gamma rays in the scintillations detector the same periodic character. The periodicity of these signals is explained by the ISS passage through the northern and southern circumpolar regions of the Earth's magnetosphere, where the flux of charged particles is increased. This is due to the fact that when one approaches the poles the fluxes of secondary neutrons and gamma rays both from the upper layers of the Earth's atmosphere and from the station itself increase. In addition to these periodic peaks, the enhanced counting rates are observed on all profiles when the station flies over the SAMA. As is well known, a substantial depression of the magnetic field is observed in the region of this anomaly. Therefore, in its vicinity the fluxes of charged particles from the radiation belts can reach the upper boundary of the atmosphere.

Figure 6 presents maps of averaged counting rates of neutrons in different detectors of BTN-M1 constructed based on data of the instrument operation during one year. The maps include the data for epithermal neutrons $0.4 \mathrm{eV}-1 \mathrm{MeV}$ (MD counter), fast neutrons with energies $300 \mathrm{keV}-10 \mathrm{MeV}$ (signal from stilbene $\mathrm{SC} / \mathrm{IN} / \mathrm{N}$ ), and gamma rays in the energy range $300 \mathrm{keV}-5 \mathrm{MeV}$ (scintillator CsI : $\mathrm{Tl}^{3+}$ of detector SC/OUT).

Due to the $I S S$ orbit inclination such maps can be constructed only for the latitude belt from $51^{\circ}$ south to $51^{\circ}$ north. The substantial enhancement of counting rates for neutrons and gamma rays in the SAMA region is very bright in the maps. Also remarkable are 

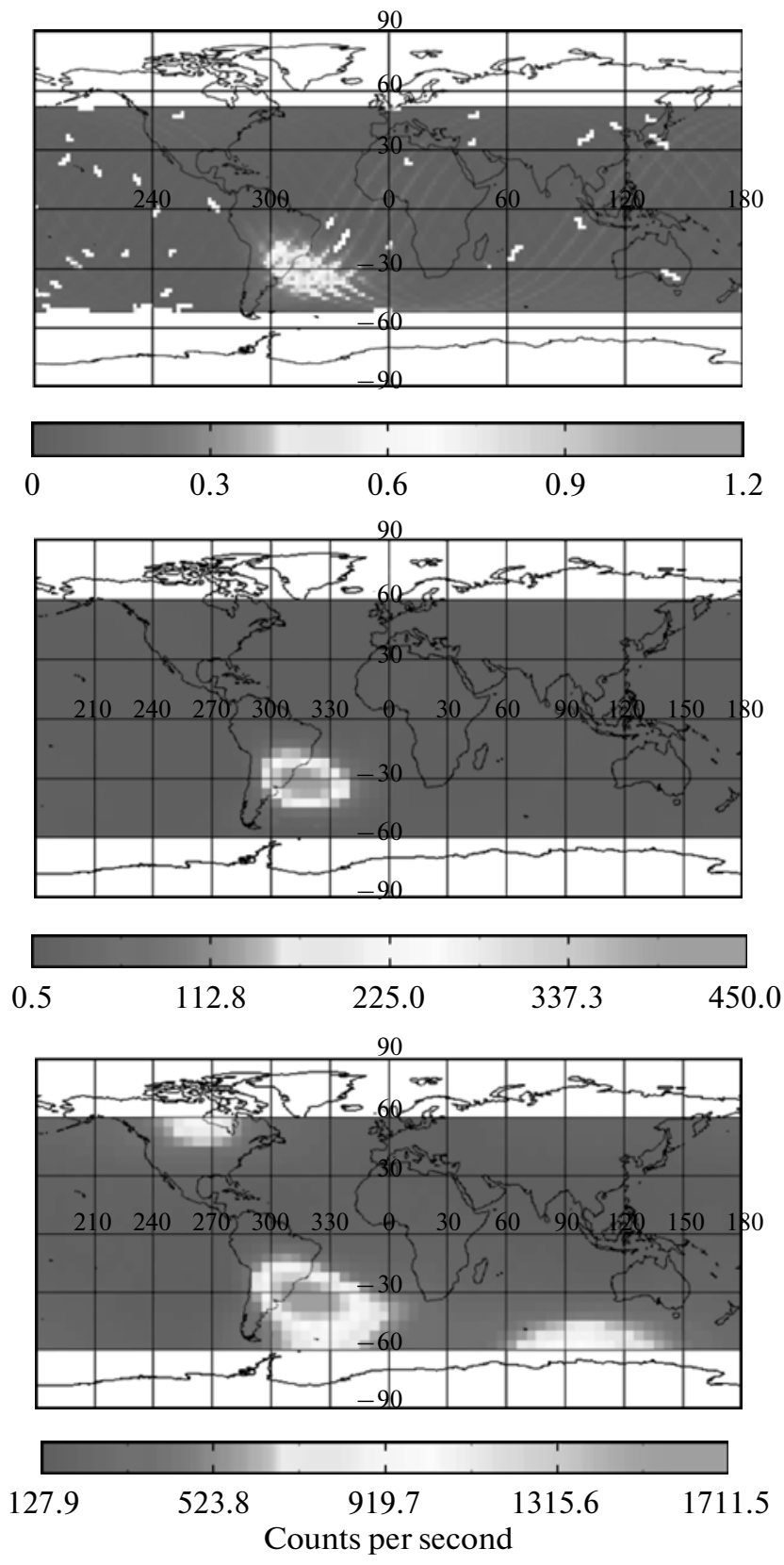

Fig. 6. Maps of averaged counting rates of epithermal neutrons in the MD detector (top map), fast neutrons in stilbene (middle map), and gamma rays in CsI: $\mathrm{Tl}^{3+}$ (bottom map). Color scales show counting rates in the detectors. Irregularities in the map for the MD detector are caused by strong noise pollution of the signal and by cutting the noise in the process of map construction.

enhanced counting rates in nearpolar regions to the south of Australia and to the north from Canada, where charged particles from the radiation belts penetrate into the upper atmosphere in the vicinity of the magnetic poles. The asymmetric picture at high latitudes is explained by inclination of the geomagnetic

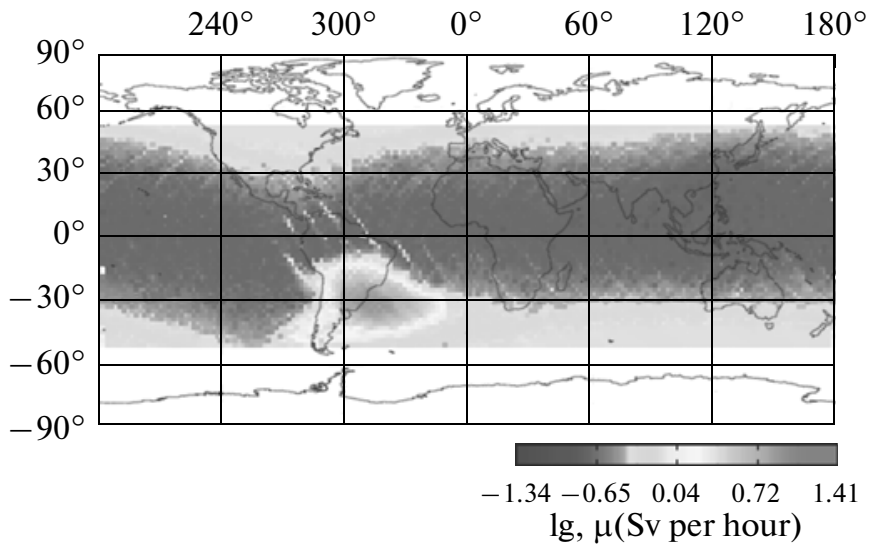

Fig. 7. A map of estimated neutron component of the radiation dose rate onboard the ISS according to the BTNNeutron data for the period February 2007-March 2008.

dipole axis to the Earth's rotation axis. A large number of passages of the station through the SAMA region allow one to make a detailed scanning of the neutron field in the region. The penetration of charged particles from the radiation belts into the atmosphere in the anomaly region is known to be intensified in the solar minimum periods, when the intensity of galactic cosmic rays in especially high in the inner region of the Solar System [19]. In 2007-2009 (at the minimum of solar activity) the contribution of SAMA into formation of the Earth's neutron albedo is quite significant. Therefore, the data of measuring the neutron radiation in the SAMA region seem to be of extreme importance: the next opportunity of such measurements will occur no earlier than in 2018.

Based on the data of neutron measurements in the energy range from the cadmium threshold of $0.4 \mathrm{eV}$ to energies of about $1 \mathrm{MeV}$, averaged estimations of the neutron component of the radiation dose rate were obtained at the place of BTN-M1 instrumentation arrangement onboard the $I S S$ for regions with different geographical coordinates along the station orbit (Fig. 7).

It is obvious that the maximum dose of neutrons was observed in the SAMA region: in 2007 the rate of neutron dose accumulation in this region was $5-6 \mu \mathrm{Sv}$ per hour, which exceeds the accumulation rate on the equator almost by a factor of 25 . Our estimations of radiation dose rate accumulation according to $\mathrm{BTN}$ M1 data are compared in Table with the results of measurements in the Japan experiment BBND [20] in a hermetic compartment of the laboratory module of the ISS American segment, and with estimated neutron component of the radiation dose obtained from the results of measurements with the HEND instrument onboard Mars Odyssey orbiting around Mars. 
Measurements of the neutron dose rate onboard the ISS and near Mars

\begin{tabular}{|c|c|c|}
\hline $\begin{array}{l}\text { Experiment, dates of measurements, } \\
\text { solar activity leve }\end{array}$ & $\begin{array}{l}\text { Measurement conditions, detectors, } \\
\text { range of measurements }\end{array}$ & $\begin{array}{c}\text { Estimation of dose rate } \\
\text { accumulation at various } \\
\text { segments of the orbit, } \\
\mu \text { Sv per hour }\end{array}$ \\
\hline $\begin{array}{l}\text { BBND experiment onboard the } I S S \text {, Mar 23, 2001- } \\
\text { Nov } 15,2001 \text {, maximum of the } 23 \mathrm{rd} \text { cycle }\end{array}$ & $\begin{array}{l}\text { Inside the } I S S, 6 \text { sensors }{ }^{3} \mathrm{He} \text { and one scintilla- } \\
\text { tion detector, } 0.03 \mathrm{eV}-15.0 \mathrm{MeV}\end{array}$ & $\begin{array}{l}3.5 \text { (equator) } \\
4.6 \text { (near-polar segments) } \\
12.0 \text { (SAMA) }\end{array}$ \\
\hline $\begin{array}{l}\text { Experiment BTN-Neutron on the } I S S, \text { Feb } 26 \text {-present } \\
\text { time, A half of the solar activity cycle }\end{array}$ & $\begin{array}{l}\text { Outside the } I S S, 3 \text { sensors }{ }^{3} \mathrm{He} \text { and one scintil- } \\
\text { lation detector, } 0.4 \mathrm{eV}-10.0 \mathrm{MeV}\end{array}$ & $\begin{array}{l}0.2 \text { (equator) } \\
0.8 \text { (near-polar segments) } \\
5.0 \text { (SAMA) }\end{array}$ \\
\hline $\begin{array}{l}\text { The HEND experiment onboard Mars Odissey, Oct 23, } \\
\text { 2001-present time, maximum of solar activity }\end{array}$ & $\begin{array}{l}\text { Outside Mars Odyssey, } 3 \text { sensors }{ }^{3} \mathrm{He} \text { and one } \\
\text { scintillation detector, } 0.4 \mathrm{eV}-10.0 \mathrm{MeV}\end{array}$ & $0.8-1.1$ \\
\hline
\end{tabular}

As a part of additional task of the experiment on detection of cosmic gamma ray bursts an analysis was made of gamma ray profiles obtained by the CsI:Tl ${ }^{3+}$ detector with time resolutions of $1 \mathrm{~s}$ and $0.25 \mathrm{~s}$. Figure 8 presents an example of detection of a gamma ray burst on December 15, 2008 by the BTN-M1 instrument and by gamma ray detectors of other spacecraft. It is well seen that variability of the luminosity profile of the gamma ray burst detected in the BTN-Neutron experiment on board the ISS is in good correspondence with those on other spacecraft.

Since neutrons are mostly produced due to interaction of GCR with the Earth's atmosphere (for BTNM1 on the ISS) and with the surface of Mars (for HEND onboard Mars Odyssey), one can estimate the GCR flux variation from variations of albedo neutrons. At the moment, the Sun is at a minimum of its activity, and, accordingly, the intensity of galactic cosmic rays is maximal [21]. Figure 9 presents the plots of increased neutron fluxes generated by GCR in orbits around Mars and around the Earth. The data of the HEND experiment were taken for the period 20022009 and for the near-equatorial region Solis Planum, where variability of neutron fluxes is caused only by variation of the GCR flux, and not by local seasonal variations of the soil composition. In the case of BTN-M1 the data are taken for the period 2008-2009, and for circumpolar $\left(45^{\circ}-51^{\circ}\right.$ north and $45^{\circ}-51^{\circ}$ south $)$ and near-equatorial $\left(15^{\circ}\right.$ south $-{ }^{\circ}$ north) regions.

The same figure presents the plot of measurements of the neutrons produced by GCR in the Earth's atmosphere according to the data of a neutron monitor at the Antarctic station McMurdo for the period 2002-2009 [22]. It is well seen that for BTN-M1 the trend is the brightest in circumpolar regions, where GCR penetrate into the atmosphere most strongly.

\section{CONCLUSIONS}

The first stage of the space experiment BTN-Neutron with instrumentation BTN-M1 allowed us in 2007-2009 to measure neutron background outside the ISS pressurized compartment and to investigate its spatial and time variations. It is planned in 2010-2012 to continue these studies when the next 24th solar activity cycle will be on the rise. Of special interest will be the data on neutron fluxes detected during solar flares, SPE, and in periods of disturbances of the Earth's magnetosphere. At the same time, the work on comparison of variations in the neutron fluxes and GCR intensity with analyzing simultaneously the data 

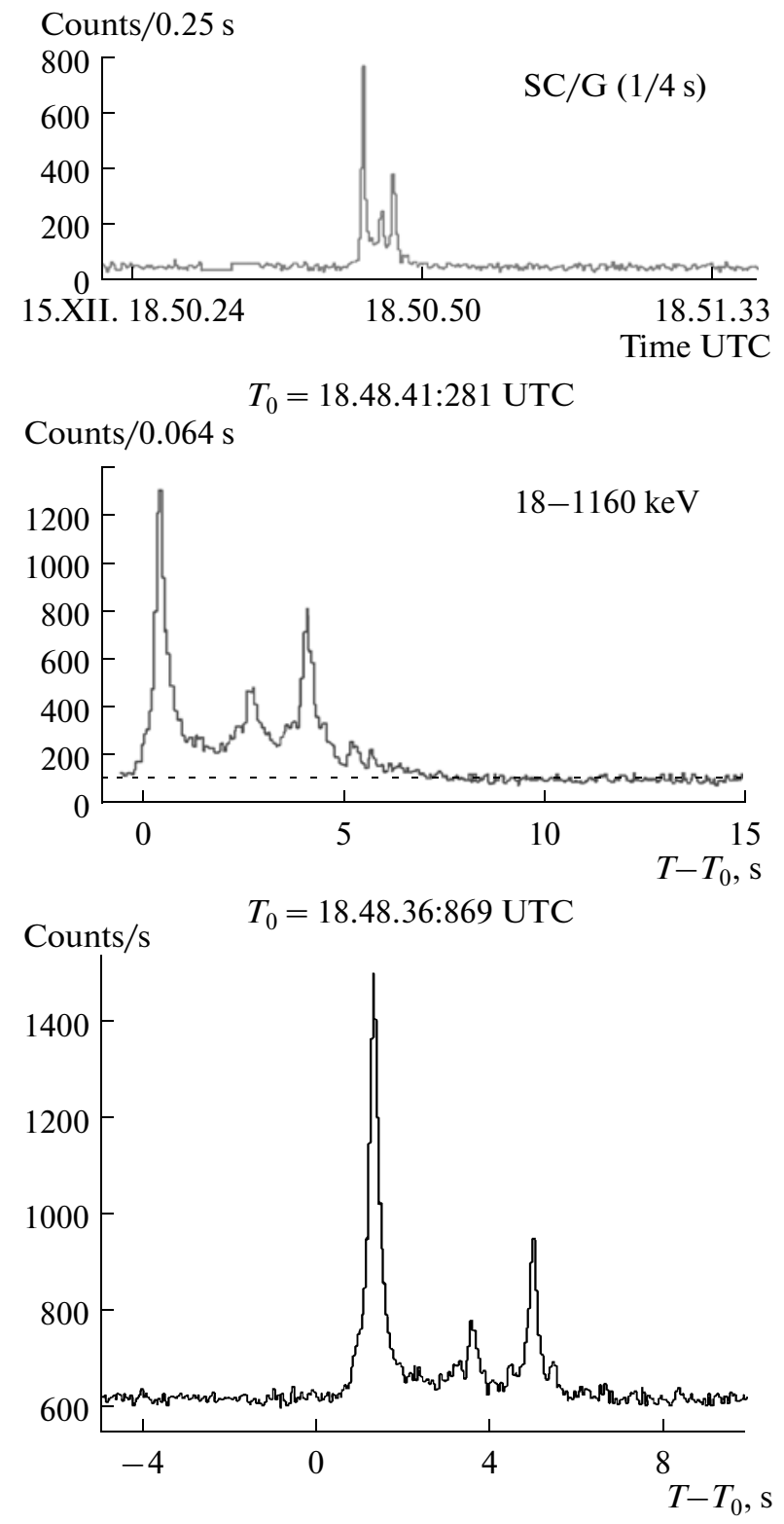

Fig. 8. An example of detection of a gamma ray burst on December 15, 2008 according to the data of BTN-M1 onboard the ISS (at the top), the KONUS instrument onboard the Wind spacecraft (in the middle), and SPLACS instrument onboard the Integral spacecraft (at the bottom).

of other space experiments and ground-based neutron monitors. After the beginning of a campaign on detection of gamma ray bursts, it is planned to include the space experiment BTN-Neutron into the international network for localization of gamma ray bursts (GCN Network, [http://gcn.gsfc.nasa.gov]) based on the method of interplanetary triangulation for determination of directions to the sources of gamma ray bursts by comparing the data from different space vehicles.

At the moment some variants are elaborated to continue the experiment with reequipment of the ISS by the set of instrumentation BTN-M2. As a prototype of the detector unit it is suggested to use the neutron and gamma-ray spectrometer NS HEND which is under design at the moment in the Space Research Institute for the Russian interplanetary automatic sample return mission Phobos-Grunt. This instrument is developed for detection of neutrons in a wide energy range from thermal energies up to energies of about 15 $\mathrm{MeV}$ and for measurement of the energy spectrum of gamma rays in the energy range from $100 \mathrm{keV}$ up to 10 $\mathrm{MeV}$. The new advanced crystal scintillator on the basis of $\mathrm{LaBr}_{3}$ is used in this instrument for detection 


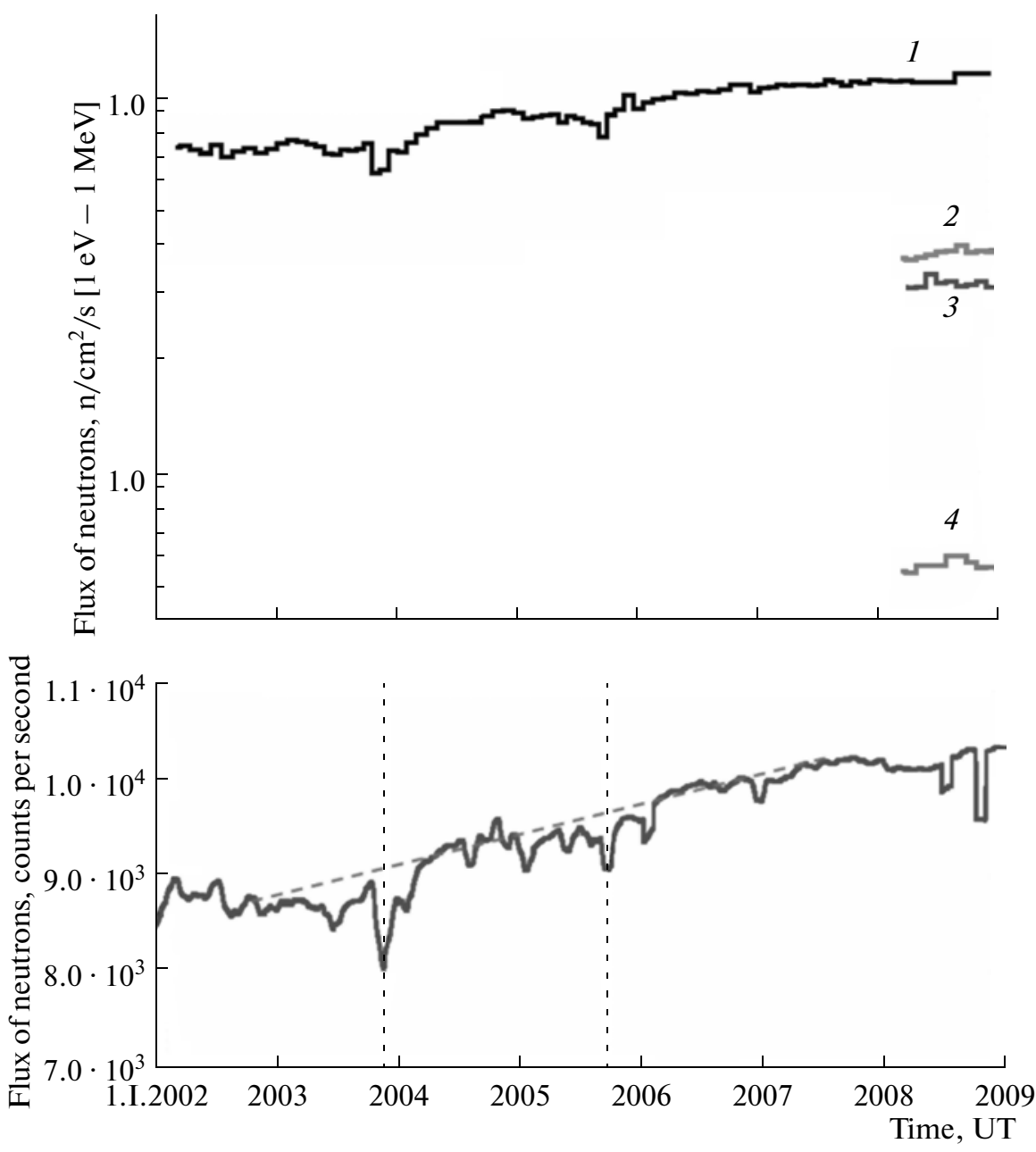

Fig. 9. Trends of neutron fluxes produced by GCR according to the HEND instrument data taken onboard Mars Odyssey (curve 1 in the upper plot) and as measured by BTN-M1 onboard the ISS (curves 2, 3, and 4 are for the southern, northern, and equatorial region, respectively). The lower plot presents the neutron flux variation according to the data of a neutron monitor of the American Antarctic station McMurdo.

of gamma rays. Radiation resistance of the samples of this scintillator was tested under space flight conditions at the first stage of the BTN-Neutron experiment in accordance with the program of expeditions $I S S$ - 14 and ISS-15 [23]. This scintillator has uniquely high spectral resolution (about 3\% for energy of $662 \mathrm{keV}$ ). It will give a possibility of measuring basic lines of the gamma ray background both from the upper atmosphere of the Earth and from ISS construction elements. These data will allow one to separate the components of neutron radiation of the atmosphere and ISS materials in the common neutron field around the station.

The second stage of the experiment is planned to be started in 2012-2013. The BTN-M2 instrumentation will be arranged in the pressurized compartments of the multi-purpose laboratory module (MLM) of the
ISS RS. It will include also a set of shields against neutrons based on hydrogenous and boron-containing compounds. The efficiency of these shields will be tested onboard the ISS. Comparison of counting rates in the detectors for different variants and directions of shielding will allow one to estimate the spatial and angular distribution of the neutron background radiation in the MLM hermetic compartment. Joint processing of measurements made by BTN-M1 and BTN-M2 instruments inside and outside the ISS pressurized compartments will allow one to construct a three-dimensional model of the neutron and gammaray components of the radiation background. Fullscale tests of variants of the radiation protection against neutrons onboard the spacecraft will be performed in order to elaborate source data for creation of 
onboard radiation shelters for the crew of long-term lunar and interplanetary missions.

\section{REFERENCES}

1. Reitz, G., European Dosimetry Activities for the ISS, Physica Medica, 2001, vol. XVII, (Suppl. 1), pp. 283286.

2. Benton, E.R., Benton, E.V., and Frank, A.L., Passive Dosimetry aboard the MIR Orbital Station: External Measurements, Radiat. Meas., 2002, vol. 35, pp. 457471.

3. Palfalvi, J.K., Akatov, Yu., et al., Cosmic Particle Induced Reaction Detection with SSNTD Stacks Exposed on-board of the International Space Station, 10th Intern. Conf. on Nuclear Reaction Mechanisms, Varenna, Italy, June 9-13, 2003.

4. Vana, N., Schoner, W., Fugger, M., and Akatov, Yu., DOSIMIR-Radiation Measurements inside the Soviet Space Station MIR-First Results, International Space Year Conference, Munich, 1992, p. 193.

5. Beaujean, R., Burmeister, S., Petersen, F., and Reitz, G., DOSTEL Dosimetry Data as a Part of DOSMAP, 7th Workshop on Radiation Monitoring for the International Space Station, Paris, France, 2-4 September, 2002.

6. Mitrofanov, I.G., Anfimov, D.S., Kozyrev, A.S., et al., Maps of Subsurface Hydrogen from High Energy Neutron Detector, Science, 2002, vol., 297, pp. 78-81.

7. Litvak, M.L., Mitrofanov, I.G., Kozyrev, A.S., et al., Seasonal Variations of Neutron Flux in the Martian Cap Regions according to Data of the Russian Instrument HEND of the NASA 2001 Mars Odyssey Mission,, Astron. Vestn., 2003, vol. 37, no. 5, pp. 413-422.

8. Nymmik, R.A., A Model of Particle Fluxes and Energy Spectra of Solar Cosmic Rays, Kosm. Issled., 1993, vol. 31, no. 6, pp. 51-59.

9. Panasyuk, M.I., Bogomolov, A.V., Bogomolov, V.V., et al., Background Fluxes of Neutrons in Near Space: Experimental Results of SINP, Preprint of Skobeltsyn Inst. of Nuclear Phys., Moscow State Univ., Moscow, 2000, no. 9/613.

10. Sevast'yanov, V.D., Tarnovskii, G.B., and Lyagushin, V.I., Measurement of the Neutron Energy Spectrum on the Mir Orbital Station, Kosm. Issled., 1997, vol. 35, no. 2, pp. 216-220. [Cosmic Research, pp. 201-205].

11. Ramaty, R. and Mandzhavidze, N., Theoretical Models for High-Energy Solar Flare Emission, in Proc. AIP Conference "High Energy Solar Phenomena. New Era of
Spacecraft Measurements"., Ryan, J.M. and Vestrand, W.T., Eds., New York: American Institute of Physics, 1994.

12. Chupp, E.L., Forrest, D.J., Ryan, J., et al., A Direct Observation of Solar Neutrons Following the 01:18 UT Flare on 1980 June 21, Astrophys. J., 1982, vol. 263, pp. 95-99.

13. Chupp, E.L., Solar Neutron Observations and Their Relation to Solar Flare Acceleration Problems, Solar Physics, 1988, vol. 118, pp. 137-154.

14. Kuznetzov, S.N., Kudela, K., Myagkova, I.N., et al., First Experience with SONG-M Measurements on Board CORONAS-F Satellite, Indian J. Radio Space Physics, 2004, vol. 33, pp. 353-357.

15. Watanabe, K., et al., Solar Neutron Events of 2003 October November, The Astrophys. J., 2006, vol. 636, pp. 1135-1144.

16. Bogomolov, A.V., Britvich, G.I., Myagkova, I.N., and Ryumin, S.P., Identification of Neutrons against the Background of Gamma Rays when Recording Them by Detectors Based on CsI:Tl ${ }^{+3}$, Prib. Tekh. Eksp., 1996, vol. 39, no. 1, pp. 13-19.

17. Bogomolov, A.V., Gudima, K.K., Myagkova, I.N., et al., Reconstruction of Neutron Spectra from Data Obtained with the Use of Scintillation Detectors Based on CsI:T1 ${ }^{+3}$, Vestn. Mosk. Cos. Univ., Ser. 3: Fiz., Astron., 1994, vol. 35, no. 3, p. 81.

18. Mokrousov, M.I. et al., Instrumentation BTN-M1 for Space Experiment BTN-Neutron on the Russian Segment of the International Space Station. In press.

19. Krymskii, G.F., Cosmic Rays and near-Earth Space, Solnechno-Zemnaya Fizika, 2002, no. 2, pp. 42-45.

20. Chishiki, A., Matsumoto, H., Koshiishi, H., et al., Analysis of the Neutron Radiation Environment inside the International Space Station as Obtained by a Bonner Ball Neutron Detector, 2nd Intern. Workshop on Space Radiation Research, Nara, Japan, 11-15 March, 2002.

21. Ishkov, V.N., The Current 23 Cycle of Solar Activity: Its Evolution and Principal Features, Proceedings of ISCS "Solar Variability as an Input to the Earth's Environment, ESA SP-535, Sept. 2003, p. 103-104.

22. Bieber, J.W., Clem, J., et al., Long-Term Decline of South Pole Neutron Rates, J. Geophys. Res., 2007, vol. 112.

23. Owens, A., The Effect of Long Term Radiation Exposure on Inorganic Scintillator Flown on the International Space Station, Nuclear Instr. and Methods in Physics Research. In press.

COSMIC RESEARCH Vol. $48 \quad$ No. $4 \quad 2010$ 